\title{
On ne parle jamais qu'une seule langue... (oui mais) On ne parle jamais une seule langue
}

\author{
Martine Delvaux
}

\begin{abstract}
How can one speak and write about oneself when the language owned is not one's own? In order to answer this question, this article follows three tracks: first, Jacques Derrida's testimony on his relation to language and accents in Monolingualism of the Other; second, Nancy Huston and Leila Sebbar's correspondance on exile; third, my own trajectory inside of the French language and the Québécois accent.
\end{abstract}

Le rapport à la langue est une histoire d'amour, une passion dévastatrice. Combien de fois entendons-nous ces questions qu'on pose à des gens dont on sait ou soupçonne qu'ils vivent dans plus d'une langue: «Dans quelle langue rêvez-vous? Dans quelle langue faites-vous l'amour?» Comment répondre à ces questions quand on souffre et jouit du monolinguisme de l'autre, qu'on est détenteur d'une langue unique que, paradoxalement, on ne possède pas? Comment y répondre quand on est bilingue, bariolé de langues auxquelles on ne s'identifie pas?

Dans Le Monolinguisme de l'autre, son essai sur son rapport à la langue française - la langue coloniale qu'il a apprise, qu'il parle comme une monolangue mais dont il dit qu'elle n'est pas la sienne, qu'elle lui a été à la fois donnée et interdite - Jacques Derrida se sert du verbe «amadouer» (aimer en enflammant) pour exprimer ce qu'on doit faire à une langue pour qu'il lui arrive quelque chose, pour qu'apparaisse sous son vêtement quelque chose comme un tatouage «où le sang se mêle à l'encre pour en faire voir de toutes les couleurs» $(1996,86)$, pour faire apparaître la langue sous les couleurs de la flamme en l'amadouant, en la faisant brûler des feux de la passions pour la marquer et la transformer, faire monter une flamme comme une autre langue dans cette langue dont il dit qu'elle est et qu'elle n'est pas la sienne.

Le monolingue (celui qui parle une seule langue qui n'est pas la sienne parce qu'elle est celle du colonisateur), nous dit Derrida, parce qu'il possède une langue, une seule, mais qu'il ne la possède pas, est projeté dans la traduction absolue, «une traduction sans pôle de référence, sans langue originaire, sans langue de départ. Il n'y a pour lui que des langues d'arrivée» (117). L'écriture, dans ce cas-ci, et pour Derrida lui-même, a pour but l'invention d'une avant-première langue qui ne pourrait pas traduire la mémoire de ce qui n'a pas eu lieu, de ce qui a été interdit - l'appartenance à une langue maternelle -, mais qui a néanmoins laissé des traces sensibles à la manière d'un membre-fantôme (118) qui ne peut jamais être réincarné; avant-première langue qui ne peut jamais exister car elle ne peut être qu'une langue promise, «une langue de l'autre, encore, mais tout 
autre que la langue de l'autre comme langue de maître ou du colon» (119); une avant-première langue, enfin, qui doit échapper au lieu de la langue comme le lieu du pouvoir et de la maîtrise. Derrida nous dit que c'est de l'intérieur de la monolangue qu'il faut inventer l'avant-première langue et ainsi montrer en quoi, si on ne parle jamais qu'une seule langue, on ne parle jamais une seule langue (123). C'est cette langue, la monolangue de l'autre, qu'il faut aimer en lui mettant le feu aux poudres, en la faisant brûler.

Je veux parler de cette histoire d'amour qu'on peut avoir avec une langue, que j'ai avec la mienne, une histoire d'amour bien compliquée, bien passionnée avec la langue française. Je veux parler de colonisation, de ce rapport à un autre qui vient $m$ 'imposer sa culture, sa langue, qui me la fait tomber sur la tête comme le coup de massue d'un dictionnaire sans que ça ne laisse vraiment de traces (quelque chose comme la technique du bottin téléphonique dans les postes de police), cette colonisation insidieuse qui est celle de la langue, douce colonisation de cette douce France que chante Trenet, de cet autre qui me dit: «Tu ne parleras pas comme ça, tu parleras comme moi.» Je veux en parler, je connais bien ça, je suis Québécoise, tout de même! Mais qui est prêt à la recevoir, cette histoire (pour une énième fois), mon éternelle lamentation autour d'une langue qui est ma passion, une langue qu'à la fois j'aime et je hais, qui m'aura autant fait jouir que souffrir? Qui peut l'entendre, cette histoire, et dans quelle langue?

Au début de cette conférence que Derrida a donnée sur la langue et qu'il a publiée sous la forme de l'essai intitulé Le Monolinguisme de l'autre, il met en scène un dialogue avec un autre, un second, une sorte d'alter ego qui lui retourne la balle, lui rend la pareille dans une joute verbale qui porte sur la langue, sur la propriété d'une langue. «Imagine-toi, dit-il à celui-ci, quelqu'un qui cultiverait le français et que le français cultiverait, un citoyen français pour qui la langue française serait la demeure, et qui viendrait un jour te dire, en bon français, "Je n'ai qu'une langue, ce n'est pas la mienne"» (13). Car cette langue qui est la sienne, le français, à lui, citoyen français (franco-maghrébin et juif d'origine), est et n'est pas sa langue, l'est totalement, absolument, et en même temps ne l'est pas du tout, et ce à jamais. "Cette seule langue, vois-tu, jamais ce ne sera la mienne. Jamais elle ne le fut en vérité» (14). Cette langue - la langue française - dans laquelle il a toujours vécu, cette langue originaire qui le traverse de bord en bord, c'est au bord de celle-ci que Derrida dit avoir vécu, c'est

[...] sur la ligne introuvable de sa côte que, depuis toujours, à demeure, je me demande si on peut aimer, jouir, prier, crever de douleur ou crever tout court dans une autre langue ou sans rien en dire à personne, sans parler même. Mais avant tout et de surcroît, voici le double tranchant d'une lame aiguë que je voulais te confier presque sans mot dire, je souffre et je jouis de ceci que je te dis dans notre langue dite commune: «Oui, je n'ai qu'une langue, or ce n'est pas la mienne.» (14) 
À cette sentence, «je n'ai qu'une langue, or ce n'est pas la mienne», l'autre, l'interlocuteur, lui répond que ce qu'il dit n'a pas de sens. «Tu dis l'impossible», dit-il. «Ton discours ne tient pas debout» (15). Car comment peut-on à la fois posséder et ne pas posséder sa propre langue? Qui plus est: comment peuton dire, en français, que le français est une langue qui à la fois est et n'est pas la sienne puisque, ce faisant, le geste performatif de l'énonciation viendrait prouver le contraire de ce qu'on impute au témoignage, à savoir une certaine vérité (15)? Et de fait, comment dire cette vérité?

L'énoncé paradoxal, contradictoire, se trouve au cœur du dialogue, à la fois fictif et vrai (puisqu'il s'adresse aussi à nous, lecteurs), que Derrida entretient avec l'autre, comme il se situe au cœur du problème que présente la langue pour Derrida. Car pour l'enfant qu'il était, franco-magrébin et juif de naissance, né et élevé en français en tant que citoyen français dans l'Algérie française, il était implicitement interdit d'apprendre ou d'utiliser l'arabe, le berbère ou l'hébreu, tout comme, malgré la permission explicite d'apprendre et de parler le français, l'obligation même de le faire, la langue du colonisateur demeurait interdite, reliée à un monde étranger dont les Franco-Maghrébins, et qui plus est juifs, étaient exclus (sous l'Occupation, les juifs d'Algérie ont vu leur citoyenneté retirée ${ }^{\mathrm{i}}$ ). La langue française était la marâtre, la langue qui brandissait la gifle sur l'accent. Le français était la loi, une loi permissive (puisqu'en apparence elle n'interdisait rien) impossible à briser, et parce qu'impossible à briser, une loi, une langue qui n'était pas vraiment celle du sujet qui la parlait, même s'il la parlait uniquement, comme sa monolangue maternelle.

Quel impact un rapport à la langue dite maternelle comme celui que décrit Derrida a-t-il sur l'identité? "Être franco-maghrébin, l'être "comme moi", ce n'est pas, pas surtout, surtout pas, un surcroît ou une richesse d'identités, d'attributs ou de noms. Cela trahirait plutôt, d'abord, un trouble de l'identité» (32). Et ce trouble, demande le philosophe,

[e]st-ce qu'il aiguise le désir de mémoire ou désespère le phantasme généalogique? Est-ce qu'il réprime, refoule ou libère? Tout à la fois sans doute et ce serait là une autre version, l'autre versant de la contradiction qui nous mit en mouvement. Et nous fait courir à perdre haleine ou à perdre la tête. (37)

Le «trouble de l'identité» que Derrida décrit a-t-il pour effet de favoriser ou d'empêcher le souvenir, de permettre ou d'empêcher l'anamnèse et donc aussi l'autobiographie? Le discours autobiographique comme le témoignage repose sur l'exercice d'une anamnèse, d'un retour sur soi, qui passe d'abord par l'identification: par cette identité nécessaire du je que doit savoir dire celui ou celle qui écrit (53). «Il faut déjà savoir dans quelle langue je se dit, je me dis» (54) écrit Derrida, demandant ainsi: 
Dans quelle langue écrire des mémoires dès lors qu'il n'y a pas eu de langue maternelle autorisée? Comment dire un "je me rappelle" qui vaille quand il faut inventer et sa langue et son je, les inventer en même temps, par-delà ce déferlement d'amnésie qu'a déchaîné le double interdit? (57)

Double interdit qui se cachait derrière une double permission apparente: s'il fallait parler le français, le français demeurait toujours la langue de l'autre à qui on ne pouvait pas s'identifier; de plus, si on avait le droit formel de parler l'arabe ou l'hébreu, c'était au même titre que l'allemand ou l'espagnol, par exemple, c'est-à-dire comme des langues étrangères. Déferlement d'amnésie comme le déferlement d'une vague, mouvement dynamique, puissant, va-et-vient d'une mer qui «porte tout», qui «remporte, rapporte, déporte et se gonfle encore de ce qu'elle arrache» (58). Comment, donc, écrire en français, dans cette langue qui est et n'est pas la sienne, langue à la fois permise et défendue? Comment «orienter» l'écriture de soi dans cette langue qui n'est pas une langue maternelle, langue qui, si elle est la sienne, est aussi celle qui aura blessé l'homme, qui l'aura traumatisé et rendu témoin de blessures et de traumatismes infligés à d'autres? «Voilà ma culture», écrit-il, «elle m'a appris les désastres vers lesquels une invocation incantatoire de la langue maternelle aura précipité les hommes» (61).

Blessures, traumatismes, lésions, scissions, écarts, traits d'union, déferlement d'amnésie, troubles... autant de symptômes, d'effets de cette mer qui se tient entre les langues: entre la langue apprise et la langue parlée, entre le français et l'arabe, entre la France et l'Algérie. Cette mer qui bouge au centre de l'identité, fait courir, fait perdre l'haleine et la tête. Cette mer donne le mal de mer comme ce fut le cas lors de la traversée en bateau que Derrida raconte avoir faite, à l'âge de dix-neuf ans, pour se rendre de l'Algérie à la France: «Premier voyage, première traversée de ma vie, vingt heures de mal de mer et de vomissements» (75). C'est ce mal qui demeure, qui marque la demeure qu'est et n'est pas la langue française devenue le lieu du malaise à cause de l'accent qui trahit et dont Derrida avoue qu'il lui en reste quelque chose (même si «on n'entrait dans la littérature française qu'en perdant son accent» [77]) et qu'il n'en est pas fier, que cet accent lui parait incompatible avec «la dignité intellectuelle d'une parole publique» (78). Accent que devait contenir l'écluse que constitue l'éducation française reçue en Algérie, l'éducation coloniale, mais qui malgré tout s'échappe, déborde, comme la pulsion d'une mer qui ferait sauter un barrage; accent qui demeure malgré tout et qui devient le lieu de la honte, de la honte qu'il y a à avoir un accent dans la perspective de la culture coloniale, et de la honte qu'il y a à avoir honte de cela.

Quel espace d'identification reste-t-il face à tant d'interdits? Où le sujet peut-il respirer, «aimer, jouir, prier, crever de douleur»? Quelle demeure demeure? La communauté traumatisée des Juifs français d'Algérie à qui on a retiré la citoyenneté durant l'Occupation, et dont Derrida faisait partie, lui sert de pôle 
pour explorer ces interdictions. Cette communauté trois fois coupée: d'abord de la langue et de la culture arabe ou berbère; ensuite, de la langue et de la culture française; enfin, de la mémoire juive. Face à cette triple rupture, dans un tel contexte, demande Derrida, «où se trouver? À qui peut-on encore s'identifier pour affirmer sa propre identité et se raconter sa propre histoire? À qui la raconter, d'abord?» (96) Comment admettre l'impossibilité d'habiter sa propre langue, la monolangue jalousement gardée qui pourtant n'est pas un «chez soi», un foyer? Comment croire que cette langue, qui est la sienne propre, «reste encore muette pour celui qui l'habite et qu'elle habite au plus proche»,

qu'elle demeure lointaine, hétérogène, inhabitable et déserte? Déserte comme un désert dans lequel il faut pousser, faire pousser, construire, projeter jusqu'à l'idée d'une route et la trace d'un retour, une autre langue encore? (109)

Derrida se bat contre ce monolinguisme qui correspond à la métaphysique qu'il oppose depuis toujours à sa pensée, la pensée de la différance, la déconstruction qui, elle, s'en prend au corps de la langue, de $s a$ langue, qui fait apparaître la langue sous les couleurs de la flamme en la faisant brûler des feux de la passion. Autant de feux, mille feux dont brûle cette langue venue d'outremer, cette langue qui est une houle, un déferlement, au cœur de l'identité.

Mais comment, dans de telles circonstances, parler «dans cette langue même comme la langue de l'autre» (42)? Comment écrire? Comment parler de soi, écrire sur soi? Comment témoigner?

Derrida parle de la passion qu'il vit en rapport à la langue, de ce rapport passionnel d'une vie-mort, jouissance-douleur dans sa propre langue, lui qui se décrit comme un martyre (cette figure par excellence du masochisme) qui vient ici témoigner de ses souffrances.

En quoi la passion d'un martyre franco-maghrébin peut-elle donc témoigner de cette destinée universelle qui nous assigne à une seule langue mais en nous interdisant de nous l'approprier, telle interdiction se liant à l'essence même de la langue ou plutôt de l'écriture $[\ldots]$ ? (51)

Et comment est-ce que je peux, à mon tour, vous raconter à vous qui occupez ici la place de cet interlocuteur, ma propre passion? Comment dire ce trouble que suscite le fait de parler une langue qui est et n'est pas la mienne? Je ne dirais pas, 
comme Derrida, qu'elle ne l'est pas du tout. Mais je vous dirais certainement que quand je la parle, je suis déjà ailleurs que chez elle, que si je m'y trouve, à demeure, que si elle est ma seule demeure, elle est aussi un ailleurs. Je ne la touche pas. Non, je n'y arrive pas. Et ça, si on peut le comprendre, dans quelle langue est-ce que je peux vraiment le dire? N'est-ce pas justement ce qui échappe à la langue, ce qui lui est incompréhensible, comme cette jouissance-souffrance de la passion du martyre? Comment dire cette vérité, dans ma langue, de cet amour-haine que j'ai pour cette langue? Comment en témoigner?

$\mathrm{Au}$ problème que pose le discours sur soi dans une langue qui est et n'est pas la sienne, Derrida répond qu' «il faudrait se constituer soi-même, il faudrait pouvoir s'inventer sans modèle et sans destinataire assuré» (96), non pas dans ce qui pourrait se présenter comme une sorte d'onanisme, mais dans le flou d'un rapport, à l'intérieur du trouble, au coeur même de ce que Jean-Luc Nancy appelle l'«il y a» du rapport sexuel qui est le lieu du rapport comme non-rapport. Le sexuel expose le rapport en tant que tel, en tant que ce qu'il n'y a pas, ce qui n'arrive jamais (angoisse que la pornographie sert à calmer en donnant l'illusion du «rapport sexuel en tant que tel», comme ce qui arrive, aboutit). Le rapport sexuel, pour Nancy, ne peut ni se compter ni se conter. On ne peut pas en faire un rapport. «On baise, écrit-il,

et en baisant $[\ldots]$ on (s')imprime une brûlure de sens. La jouissance n'est rien qu'on puisse atteindre: elle est ce qui s'atteint et se consume en s'atteignant, brûlant son propre sens, c'est-à-dire l'illuminant en le calcinant. (Nancy 2001, 52)

Et il en est de même de cette langue avec laquelle je ne rentrerai jamais en rapport, que je n'aimerai jamais au sens où je l'atteindrais comme une jouissance, comme le but d'un rapport à la langue, un accomplissement ${ }^{\mathrm{ii}}$. Au contraire, il faudrait que je ne vous parle pas, en français, ou que je m'adresse à vous comme si vous n'étiez pas là, ou que je lance vers vous mes paroles mais en ratant exprès la cible, que je m'adresse à côté de vous, que je m'attende à ce que vous ne m'entendiez pas et que ce soit très bien ainsi. Il faudrait que je m'invente sans modèle et sans destinataire assuré et que je fasse brûler le sens, que je brûle de jouissance et parle quelque chose comme cette langue amadouée à laquelle Derrida fait référence, une langue que j'aimerais en la faisant brûler, en la plaçant bien haut sur un bûcher. Je te dis que je t'aime, mais je te le dis dans une langue que tu ne comprends pas, dans cette langue que j'invente, une langue qui, quand je la parle, tourne cent fois à l'intérieur de ma bouche, qui palpite, se tend et se détend, qui fait des virevoltes, des volte-faces.

Cette langue qu'on parlerait uniquement sans pour autant la posséder, cette langue qui est et n'est pas notre langue, il faudrait l'attendre comme on attend cette jouissance qui ne vient jamais (même quand on dirait qu'elle y est) et 
dont le plaisir repose justement dans l'attente. Une langue qui ne sait que se faire attendre, dont il faut s'attendre à ce qu'elle n'arrive jamais. Comme la langue que Derrida écrit (cette pratique déconstructionniste de la langue) et par laquelle il cultive l'impossibilité de traduire: la copie ne peut pas rendre compte de l'original; il n'y a pas de fidélité dans le rapport, il y a toujours un reste. Derrida avoue que ce qui l'intéresse et ce qui sert la déconstruction du phallogocentrisme occidental, c'est la traduction en français, dans sa monolangue, d'une possibilité inaccessible, une parole qu'il ne connaît pas encore, qui perpétuellement se fait attendre. Comme j'attends, illusoirement, ce moment où ma langue $m$ 'apparaîtra enfin comme la mienne et non plus comme celle de l'autre, la monolangue de l'autre que je lui ai empruntée, dont je me sers peut-être contre son gré, la petite cousine du Québec qui parle toujours bien mal la langue du grand cousin français, qui lui fait honte à cette langue qu'il lui a donnée, comme si une langue ça se donnait en tant que telle, alors que la langue n'est jamais donnée, qu'elle reste toujours à donner, comme un don possible et impossible, dont il faut en prendre et en laisser. Mais je ne veux plus penser à la langue au sein d'un rapport de pouvoir, j'en ai plein le dos de ce sentiment de victimisation. Je ne veux plus m'identifier avec les colons français ou les patriotes québécois. Et quand je parle anglais, je voudrais avoir d'autres images que celle des colonisateurs britanniques d'antan ou des impérialistes américains du présent. Je ne veux plus comparer mon accent à celui du Français, penser au Québec comme à un succédané de la France, un Nutrasweet de la langue, quelque chose d'aussi sucré mais de combien moins bon, combien moins vrai, faux-ami, faux-frère, faux-Français. Voilà ce que je veux faire brûler: tout cet enchevêtrement de racines qui sert de rapport à ma langue.

Après avoir écrit les Lettres parisiennes, la correspondance qu'elle a tenue dans les années 80 avec Leïla Sebbar sur le sujet de l'exil, Nancy Huston (auteure d'origine canadienne-anglaise qui vit, écrit et publie en France depuis de nombreuses années) a subi pendant six mois une période indolore de paralysie qu'elle décrit ainsi: «J'étais un arbre qui ne sentait plus que ses racines» (Huston 1990, 41). La correspondance, écrite en français sur le rapport au pays d'accueil qu'était la France et sur le pays d'origine qu'est le Canada, avait eu pour effet de ramener l'écrivaine à ses sources. C'est un trouble de l'identification que représente cet état paralytique comme aussi la nausée qu'elle décrit sentir au moment de l'arrivée lorsqu'elle revient au Canada (le pays trahi), les houles de nausée que Derrida a vécues quand il a traversé la Méditerranée pour passer de l'Algérie à la France, lorsqu'il a rejoint ce maître qui habite outre-mer, cette capitale qui interdit. Autant de malaises qui symbolisent les dangers d'une culture et d'une langue «maternelles» dans lesquelles on doit croire parce qu'elles s'imposent, qu'elles l'exigent, ou parce qu'on pense que ça va de soi. Le trouble provient de cette langue qu'on croit avoir, que l'on croit arrivée, qu'elle 
nous a vraiment été donnée alors qu'on nous a mis des mots dans la bouche pour nous asphyxier.

Et moi, quand j'étouffe en français, dans ma langue française, quand je ne trouve pas les mots, que la syntaxe fout le camp, part en déroute, que je m'entends parler ma langue comme une langue étrangère et que je me punis, en silence, que je m'en veux de ne pas être à la hauteur de cette sacrée langue française, ma langue française, ma langue maternelle, ma marâtre comme dirait Derrida, mon éternel geôlier... Elle me guette sans arrêt, elle me surveille comme depuis la tour d'une prison panoptique que j'ai tout à fait intégrée, que je porte désormais en moi comme la bonne petite prisonnière que je suis devenue par le biais de cet apprentissage de la langue, de la bonne langue, de la vraie. Ma langue me surveille comme ma mère surveillait ma langue quand j'étais enfant. Comme elle me corrigeait, ma mère réviseure, l'éternelle correctrice des épreuves de ma vie. Ma langue est un maitre sadique.

«Voilà ma culture», écrit Derrida, «elle m'a appris les désastres vers lesquels une invocation incantatoire de la langue maternelle aura précipité les hommes» (61). Le désastre de la langue maternelle. Quand on invoque la langue maternelle, on invoque le souhait de rester entre nous, à l'intérieur d'une consanguinité imaginée. On rêve de rester en famille. Le dialogue est intime. Il a lieu dans le partage d'une langue commune, dans l'imagination d'un même état, des mêmes racines. On regarde la forêt plutôt que l'arbre. L'arbre se perd dans la forêt. Et ensemble, on est prêt à faire voir aux autres qu'on se chauffe au bois de la langue maternelle. Et si je disais que moi, quand je me retrouve dans ma langue, avec d'autres qui la parlent, je me crois souvent dans la langue d'un autre? J'entends une langue qui n'est pas la mienne, et je sais que la mienne, ma langue, est une langue qui n'existe pas, que je l'ai même cherchée (comme Nancy Huston) dans la langue de l'autre, du vrai autre, de l'autre radical qu'est, pour un Québécois, l'Anglais. Mais si je voulais carrément y mettre le feu, moi, la Québécoise que je suis, mettre le feu à mes racines, les brûler? Si je voulais l'amadouer, cette langue de mes ancêtres, la faire brûler du feu de mes passions? Qu'est-ce que ça donnerait?

Disons donc que je ne veux plus être la bonne élève, celle qui s'efforce de bien parler français, celle qui protège cette langue au prix de la créativité, de cet état de bordel qu'il faut infliger à la langue pour la faire respirer. Disons que j'en ai assez de préserver la langue du maître, de la garder avec mon bouclier, de la défendre, car je ne lui fais pas de mal, à la langue, à ma langue. Au contraire, je la bichonne, je la minouche, je lui fais des câlins pour être certaine qu'elle m'aime bien, qu'elle me reconnaît comme digne de la parler. Une névrose que je retrouve chez Derrida qui avoue son intolérance aux accents des autres (au mien, par exemple) et au sien, à cette inflexion de la voix qui resurgit quand il perd le contrôle, que le barrage du verbe et de la voix cède et que le ton monte, ce ton qu'il avait dans sa famille et qui le dénonce comme celui qui n'est pas tout à fait Français. Cette honte de ne pas être l'autre dont on parle la langue. Et cette honte qu'il y a à avoir honte de ça. 
Dans Désirs et réalités, Nancy Huston raconte l'altercation qu'elle a eue avec une Française après une conférence donnée en France sur sa désidentification d'avec son pays d'accueil et au cours de laquelle elle répétait, comme un leitmotiv, au début de chaque paragraphe, les mots «J'ai honte de la France» (honte de la France pour sa politique nucléaire, pour son provincialisme obstiné en ce qui concerne les revendications des femmes, honte de la France pour ses attitudes de supériorité, etc.). À la fin de sa présentation, une femme (française) s'approche d'elle et lui dit: «Je crains que vous n'ayez pas une maîtrise suffisante de la langue française pour bien mesurer l'impact du mot "honte"». «Mais Madame! je suis Française!» lui répond Huston. Et la bonne femme de répliquer: «Hum! Vous n'êtes qu'une pièce rapportée! Moi, je suis Française depuis cinq siècles!» $(1999,92)$. C'est ça, le colonialisme, dans sa forme la plus ordinaire mais aussi la plus insidieuse: le besoin qu'a l'autre de se nommer propriétaire d'une langue pour pouvoir l'interdire. C'est pour mieux te manger, mon enfant! C'est ça, le monolinguisme de l'autre: une langue d'ores et déjà interdite par le maître mais la seule qu'on peut parler et appeler "sa propre langue», une langue qu'on ne peut parler qu'en ayant honte de le faire.

Huston et Sebbar, par leur correspondance, cette communauté qu'elles créent de femmes exilées dans la langue de l'autre, cherchent une porte de sortie à la honte. Leur exil nomme cette difficulté, pour une femme en particulier, d'adopter la langue maternelle pour écrire; car pour écrire, il lui faut nécessairement à la fois adopter et renier la langue, l'aimer et la haïr, la blesser et lui procurer du plaisir, mais dans tous les cas, refuser de faire corps avec elle $e^{i i i}$. Dans le choix amoureux et douloureux qu'elles font de s'exiler dans la langue - Sebbar en refusant d'apprendre l'arabe et d'écrire en français, Huston en choisissant de vivre et d'écrire en français plutôt qu'en anglais ${ }^{i v}$-, il y a un refus de la langue maternelle comme de ce en quoi on peut croire, de ce qui est arrivé, de ce qui nous a donné la vie. La vie comme la langue, comme la jouissance, qui resterait toujours à être donnée. C'est ce qu'indique aussi le choix d'écrire et de publier cette correspondance. Le soi devient étranger par le monolinguisme de l'autre. Liberté de l'échange épistolaire qui laisse la place à l'invention de soi et de l'autre comme à l'invention de la langue: d'une langue qui n'est plus une monolangue mais une langue à deux, qui vient toujours de l'autre, ce qui correspond à l'éthique qu e Huston suggère dans Nord perdu: «L'étrangéité est une métaphore du respect que l'on doit à l'autre. Nous sommes deux, chacun de nous, au moins deux, il s'agit de le savoir!» (37) Et n'est-ce pas là la condition du rapport sexuel et amoureux?

Au bout du compte, au terme de la fable que propose Derrida dans $L e$ Monolinguisme de l'autre et qui est son histoire, le rapport à la langue a à voir avec la possibilité qu'a le sujet de parler de soi, de témoigner sur lui-même au sein 
de cette logique paradoxale qui caractérise le témoignage comme elle caractérise le monolinguisme de l'autre. "Tu dis l'impossible» répond l'interlocuteur i $\mathrm{m}$ a g inaire de Derrida, nommant la possibilité impossible de l'acte qui consiste non seulement à dire qu'on n'a qu'une langue et qu'elle n'est pas à soi, mais la possibilité impossible du témoignage lui-même. Et pourtant, c'est bien un témoignage que propose Derrida, témoignage sur «l'origine de [s]es souffrances» (14), témoignage du martyre juif franco-maghrébin qui demande à son interlocuteur de le croire sur parole, de croire l'incroyable de ce rapport particulier à la langue qu'il lui demande de recevoir comme exemplaire, c'est-à-dire comme universel. C'est bien là le propre du témoignage que de témoigner de l'incroyable, de ce qui ne peut qu'être cru car on est le seul à l'avoir vécu, et en même temps de faire état d'un universel, de ce qui est généralisable parce que répétable, toujours déjà répété. Et l'expérience de la langue, demande Derrida, «[n]'est-ce pas ce qui donne lieu à cette articulation entre l'universalité transcendantale ou ontologique et la singularité exemplaire ou témoignante de l'existence martyrisée» (50)?

En quoi la passion d'un martyre franco-maghrébin peut-elle donc témoigner de cette destinée universelle qui nous assigne à une seule langue mais en nous interdisant de nous l'approprier, telle interdiction se liant à l'essence même de la langue ou plutôt de l'écriture, de la marque, du pli de la re-marque? (51)

Dans Demeure, son essai sur la question du témoignage, Derrida propose une phrase comme exemplaire de l'acte de parole testimonial: «À l'instant, à cet instant même, je parle français, nous parlons français. Voilà», écrit-il, «un témoignage» $(1998,37)$. Cet énoncé est un témoignage exemplaire: d'abord, un je y prend la parole; ensuite, il la prend pour décrire une situation, un état, pour dire qu'il parle français; enfin, cette prise de parole constitue un acte présent on ne pourrait pas dire, en français, «à l'instant, à cet instant même, je parle anglais». De même, on ne pourrait pas dire cet énoncé en anglais, on ne pourrait pas le traduire (car il ne serait plus un acte, un témoignage). Le témoignage, comme la monolangue, est intraduisible: il est impossible de le traduire directement, de le faire simplement passer d'une langue à une autre. Comme la monolangue, le témoignage (dont on croirait qu'il est la forme la plus pure, la plus directe du dire autobiographique) est le lieu d'une traduction absolue, jamais finie. On pourrait dire du témoignage, comme de la monolangue, qu'il est spectral, à la fois dire et indicible, mémoire et absence de mémoire, vérité et fiction, comme cette monolangue dont Derrida dit qu'à la fois elle est et n'est pas la sienne.

Spectralité de la monolangue qu'il aura sans cesse cherchée autour de lui et dont il aura partout repéré les marques, qu'il aura re-marquée entre autres dans le témoignage. D'où l'importance de l'échange mis en scène au début de l'essai. 
Cet échange est et n'en est pas un: il n'en est pas un puisqu'il est fictif, mais il en est un dans la mesure où on y assiste, nous lecteurs, tiers à la fois inclus et exclus, compris dans la figure de cet autre à qui Derrida s'adresse mais situés à l'extérieur de ce dialogue dont on ne fait pas tout à fait partie même s'il nous prend à partie. Cette mise en scène de l'échange a tout à voir avec l'accident de circulation de la langue, cet accident qui est venu court-circuiter chez Derrida son rapport à soi, à son histoire, qui est venu faire obstacle à son auto-exposition. Cet échange, cette scène testimoniale, correspond bien à la réponse qu'il donne au «où se trouver» par rapport aux interdits coloniaux de la monolangue: «Il faudrait se constituer soi-même, il faudrait pouvoir s'inventer sans modèle et sans destinataire assuré» $(1998,96)$. Il faudrait toujours déjà se constituer soi-même comme autre, dans une langue qui vient de l'autre, qui est la venue de l'autre.

Et n'est-ce pas ce à quoi correspond l'exercice de la correspondance intenté par Nancy Huston et Leîla Sebbar? L'autre à qui on écrit, celle qui reçoit les lettres, est à la fois inclue et exclue. Si le tiers est appelé à recevoir, à traduire le texte monolinguistique qui lui est donné, ce texte n'est jamais complètement donné et ne peut donc jamais être tout à fait reçu. L'échange épistolaire de Huston et Sebbar, ces Lettres parisiennes, justement parce qu'il s'agit de lettres, met en scène la traduction testimoniale à la fois impossible et absolue dont traite Derrida, l'invention de soi sans destinataire assuré. L'autre présent, nommé, à qui on s'adresse dans l'échange, n'est pas pour autant assuré. Il demeure, dans le cas de Huston et Sebbar, la figure de l'autre, de soi comme autre, la demeure que trouve le soi dans l'autre, qu'il s'agisse de la langue ou de l'ami. Comme l'écrit Huston: «On doit se répéter, mais jamais dans l'identité» (164).

Il faudrait toujours déjà se constituer soi-même comme autre, comme le suggère Derrida, dans une langue qui vient de l'autre, qui est la venue toujours attendue de l'autre. Il faudrait se concevoir à la lumière du rapport, avec quelque chose d'ouvert, d'incomplet, de jamais accompli, de jamais fermé. Et la seule façon d'y demeurer, c'est en faisant arriver à la langue non pas un enfant (c'est ce que dit Derrida), mais plutôt quelque chose comme un tatouage, le tatouage du langage, là où le sang se mêle à l'encre, où la passion à la fois exalte et sacrifie la langue et celui, ou celle, qui la parle.

Dans une note au Monolinguisme de l'autre, Derrida raconte l'histoire, tirée d'un film japonais, d'un artiste qui, en lui faisant l'amour, couvre le dos de sa femme d'un tatouage qui est son chef-d'oeuvre. Cette femme le quitte à cause de la violence de l'acte, cette passion du tatouage, et emporte avec elle leur fils qu'elle renverra seul plus tard à son père. La fin est tragique, nous dit Derrida, le film ne la raconte pas, mais il dit que seule la femme survit de ces trois personnages, ainsi que le chef-d'oeuvre qu'elle porte sur sa peau, dans son dos. Un chef-d'oeuvre qu'elle ne pourra jamais voir sinon par le biais d'un miroir. Le rapport à la langue de l'autre doit peut-être justement se faire ainsi. Comme un rapport du non-rapport, l'amadouement d'une langue qu'on se passionne à ne jamais 
posséder, dont on ne jouit vraiment jamais parce que cette jouissance est impossible et que c'est ainsi qu'il faut aimer, dans cette quête éternelle, ce geste qui consiste à regarder un tatouage qu'on porte dans son dos en se cassant le cou pour le voir à l'envers par le biais d'un miroir.

Voilà donc ce qui reste à faire: poser un geste de passion comme celui du littéraire qui sublime et sacrifie la langue. Comme l'écrit Huston à Sebbar à la fin de leur échange: «Le seul écart indispensable est celui du geste littéraire luimême. À ce moment-là, anything can happen, non?» (213). Oui, anything can happen... Je vous le dis: le rapport à la langue est une histoire d'amour, un rapport sexuel, une passion incommensurable. Je vous le dis à vous, destinataires qui ne me sont pas assurés et qui ne me rassurent pas: On ne parle jamais qu'une seule langue, on ne parle jamais une seule langue. Et peut-être ne fait-on jamais non plus l'amour qu'avec une seule personne (comme on ne ferait jamais un seul deuil à la fois). C'est là la beauté terrifiante de la passion qui brûle tout. L'amour comme la langue.

Il faudrait écrire comme on fait l'amour. Et après, quand on pense que c'est fini alors que ça ne peut que continuer, toujours, encore, encore plus, on pourrait dire quand même, comme je vous le dis maintenant, comme l'écrit Huston à Sebbar à la fin de sa dernière lettre: «Je te laisse. Je suis épuisée, et apaisée» (213).

Notes

i Si Derrida était un citoyen français, cette citoyenneté, il l'a perdue «sous l'Occupation, comme on dit» (ibid., p. 35), en tant que juif, Occupation symbolique puisqu'il n'y a pas eu d'Allemands sur le sol algérien, Occupation effectuée par les administrateurs français, ceux-là qui représentent la langue et la Métropole: la France. «Je ne doute pas que de telles exclusions viennent laisser leur marque sur cette appartenance ou non-appartenance de la langue» écrit-il (loc.cit.). Car qui en fait possède la langue? Peut-on jamais posséder une langue? Peut-elle jamais être notre demeure? «Nous fûmes otages des Français, à demeure, il m'en reste quelque chose, j'ai beau voyager beaucoup» (ibid., p. 36).

ii Derrida révèle ce fantasme qu'il porte d'une voix qu'il trouverait et qui lui révélerait la vérité de ce qu'il a vécu: «elle-même au-delà de la mémoire, comme l'envers caché des ombres, des images [...]». Comme s'il avait toujours été coupé de la réalité et que tout en lui tendait vers cette promesse irréalisable. (Ibid., p. 135.)

iii «Écrire sur du manque», dit Sebbar, «un manque fondamental [...] sur du silence, une mémoire blanche, une histoire en miettes, une communauté dispersée, éclatée, divisée à jamais, [...] sur du fragment, du vide, une terre pauvre, inculte, stérile où il faut creuser profond et loin pour mettre au jour ce qu'on aurait oublié pour toujours"». (1988, 160)

iv Née et élevée en anglais en Alberta puis sur la côte est des États-Unis, Nancy Huston a fait le choix d'immigrer en France, le pays où, depuis, elle vit et écrit d'abord et avant tout en français. Si Derrida se dit le seul franco-maghrébin de 
naissance dans la salle, Huston se dit la seule auteure française et canadienne, sans trait d'union, sans être canadienne-française. En choisissant d'abandonner sa terre et sa langue natales, Huston dit s'être propulsée dans un état d'illégitimité: «Je suis une fausse Française, une fausse Canadienne, une fausse écrivaine...» Leîla Sebbar, de père algérien et de mère française, élevée en français au sein de l'Algérie coloniale, dans les écoles françaises d'Algérie où ses parents enseignaient, s'est exilée à Paris où elle aussi vit et écrit en français, ayant toujours refusé d'apprendre l'arabe, la langue de son père. Que sa langue maternelle est la langue française, le nom de Sebbar, le nom de son père, ne le dit pas, et c'est ce paradoxe qu'elle creuse dans l'écriture.

\section{Bibliographie}

Derrida, Jacques. Le Monolinguisme de l'autre. Paris: Galilée, 1996.

- Demeure, Paris: Galilée, 1998.

Huston, Nancy. Journal de la création. Paris: Seuil, 1990.

- Nord perdu, suivi de Douze France. Arles/Montréal: Actes Sud/Leméac, 1999.

Huston, Nancy et Sebbar, Leîla. Lettres parisiennes. Histoires d'exil. Paris: Bernard Barrault/J'ai lu, 1988.

Nancy, Jean-Luc. L'il y a du rapport sexuel. Paris: Galilée, 2001. 